\title{
VISUAL ANALYSIS FOR CONCEPTUAL DESIGN OF COMPLEX SYSTEMS
}

\author{
L.D. GITELMAN, O.B. RYZHUK \& M.V. KOZHEVNIKOV \\ Department of Energy and Industrial Management Systems, Ural Federal University, Russia.
}

\begin{abstract}
Managing the development of complex organizational, technological, and socio-cultural systems calls for the construction of conceptual models that would define the properties and links between the elements of the systems and focus the attention of decision makers on the most significant aspects. The authors propose the visual analysis method as a tool for the conceptual design of complex systems. The method is based on the building of a series of visual images that are associated with the purpose sustainable development and holistic representation of the system. At the initial stage, the general structure of a complex system is analyzed and the required degree of detail of its elements is determined. The system is then broken down into elements and a specific visualization tool is selected for each of them so that the properties of the systems that need improving are highlighted. Ultimately, a conceptual design of the system is created that suggests managerial solutions that meet the interests of the stakeholders. The method has been tested in a number of projects that were implemented with the purpose of ensuring the strategic leadership of major manufacturing companies.

Keywords: complex systems, concept, conceptual design, decomposition, model, multi-project, visual analysis, visual language, visualization.
\end{abstract}

\section{INTRODUCTION}

A wave of new technologies, their convergence and integration are in the everlasting process of renovation. At the same time, interconnections between technologies and socio-economic and organizational systems are getting wider and deeper. Such systems are super-complex and require customized tools for managing their creation and operation throughout their lifecycle in which the research phase acquires particular importance. It is the scholarly investigation of complex systems that generates new hypotheses, knowledge and concepts.

As shown in [1], amid uncertainly and risk, the key success factor in forecasting the development of systems lies in anticipatory management. The adoption of anticipatory management practicies is a radically new project that constitutes a serious challenge for business as well as for education and science. Essentially, the question is about conducting an overhaul of the paradigm of development models and forging links within the smart partnership of science, education, business, and government.

The multidisciplinary nature of the smart partnership calls for constructing a common language of concepts and new communication formats [2]. Using a combination of various language systems provides a fuller set of means for conveying the meaning, compared to rather scanty capabilities of the semiotic systems of spoken and written language [3]. That's why interdisciplinary communication has generated a fundamentally new semiotic system that is called visual - verbal language. Along with other actively developing methods, there is an opportunity for the accelerated development and use of visual language for the purpose of anticipatory management. Therefore, it becomes appropriate to use visualization, which means any technique for representing data as a graphical image delivering a holistic representation of a complex subject matter, for addressing the tasks of anticipatory management at the current stage of the development of science and advanced practices in this field $[4,5]$. 
Anticipatory management puts forth new content requirements for research of complex systems [1]. The requirements pertain to the adoption of new instruments of representation of the future, design of its individual elements, first and foremost of promising markets, technologies and properties of new products and services. The issue of creating value for customers interacting with the system comes to the forefront. In the course of design, it is necessary to lay out changes in technological processes, management systems, business models, and competences. The research results in a sequence of transformations that one needs either to undertake right now or to ensure the preparedness for change when a realistic scenario of the future is identified.

Systems engineering is an advanced technique of complex systems analysis [6,7]. Its toolkit, including new research areas, has seen intense development [8-11]. Conceptual design is a starting point in the use of this technique [1]. The novelty of the proposed solution lies in the application of visualization tools for analysis of conceptual models and keeping a research log and recording research results by means of visual language. The authors suggest that visual analysis that contains the principle of the logical construction of visual images should be used as the prime instrument of conceptual design.

\section{TRANSFORMATION OF THE LANGUAGE OF CONCEPTUAL DESIGN}

The phenomenon of visualization has only recently started to be given attention in science and practice, and so has visual language that emerged as a new mode of communication on its basis. Images and visual artifacts are not just add-ons to verbal texts, mere transmitters of information, or means of communication: they have become an elementary mode for the construction, maintenance, and transformation of meaning [12].

The facticity of visuals is further enhanced by the fact that argumentation disappears. Aspects of visual artifacts are more difficult to ignore compared to aspects of verbal texts. Visuals communicate with an immediacy of reception and a memorable impression of the essence of the message - an essence that, in linear verbal texts, has to be assembled piece by piece over time. It is for these reasons that a consideration of the visual mode of discourse and meaning construction will augment the understanding of contemporary organizational practices, and that it therefore has to play a prominent role in organizational and management research [12,13].

Visual language can be defined as the tight integration of words and visual elements and as having characteristics that distinguish it from natural languages as a separate communication tool as well as a distinctive subject of research. The language was created as the merger of component visual vocabularies from such widely separate domains as engineering diagramming, visual metaphors, cartoons, quantitative graphs and charts, illustration and photography, mechanical drawing, film narrative, and animation techniques [14].

Along with examinations of the meaning and research of tools of visual language, the science of visualization has been given scholarly attention [15]. Coupled with digital tools, visual language could eliminate the main barriers to thinking and the transfer of big complex ideas among people. It effectively solves the problem of the representation and transfer of mental models, which is particularly relevant in the case of poorly structured problems.

Factors driving the development of visual representation techniques are shown in Table 1.

Consequently, the use of visualization enriches the methodology of designing conceptual models of complex systems and boils down to choosing suitable visualization instruments that are tailored to treat ideas as systemic wholes. 
Table 1: Drivers of development of visual representation techniques.

\section{Changes in data volumes}

Exponential growth of information.

Irregular data volume

New user tasks
Problem of studying huge volumes of intricately structured data

Need to solve problems with excessive or incomplete information captured in a trend or range format Unconventional filtering and detalization requests

\section{Growing complexity of systems}

Complex structure tasks

Demand for modelling

Rapid feedback to changes
Demand for supplementary instruments for task optimization and solution identification due to limitations of smart systems Visualization of processes and systems for the purpose of research and improvement

Management optimization on the basis of system status indicators

\section{Growing computational capacity}

Growing capacity of com- Growing computational capacity of CPUs, monitors. Higher munications equipment bandwidth of networks

New generation of softMathematic methods and algorithms of visualization. Adware vancement of graphics software, including those incorporating VR\&AR

Controlled interaction Dynamic feedback between control and controlled systems through visual interface

\section{Transformation of communication modes}

Natural language loses its exclusive role as transmitter of meaning Information overload Interdisciplinary teams
Growth of verbal - graphical means of information transfer

An avalanche of messages with complex concept structures Demand for unified conceptual vocabulary for experts in various domains and from different countries

\section{HANDS-ON PRACTICE WITH VISUALIZATION IN CONCEPTUAL DESIGN}

Hands-on practice with visualization is delivered with the application of conceptual design techniques that reveal various aspects of the operation of systems. The result of the experiment is a conceptual model of a complex system that includes a system of ideas and relationships among them [6].

In Table 2, the authors sum up visualization tools that are utilized for describing the key properties of complex systems.

When constructing a conceptual model of a complex system, one encounters the following typical problems:

1. identifying classes and groups of elements within the complex system and establishing links between them; 
Table 2: Visualization tools for describing key properties of complex systems.

Attribute Visualization tool

Openness that lies at the Rich picture

heart of active interaction Sketches, drawings, photographs, symbols, titles that depict a with environment situation or an issue from the perspective of a person or a group of people. It covers people, systems, processes, interfaces, information, infrastructure units, factors, emotions.

Diversity, partial autonCommunication diagram omy and links between Communication Diagram models the objects or parts of a agents system, the interactions (or messages) between them, and the sequence in which these interactions occur. A communication diagram is a combination of information, its sequence and application options. It describes both the static and dynamic behavior of the system. Communication diagrams use a free-form arrangement of objects and their interactions within a system.

Agent self-organization and absence of centralized control over agents

Indeterminate behavior of agents that implies freedom of choice and, at least, several pathways to results

Emerging properties of a self-organizing system that are absent in agents that constitute it

\section{Semantic network}

It represents a subject area as a directed graph with nodes and arcs that link nodes. Networks can cover concepts, events, properties, processes. A network reflects the semantics of the subject matter in the form of concepts and relationships. The items of a semantic network are placed into simple shapes and connected with labelled arrows.

\section{Data-flow diagram}

A data flow diagram (DFD) is a graphical representation of the "flow" of data through an information system, modelling its process aspects. It shows what kind of information will be input to and output from the system, how the data will advance through the system, and where the data will be stored. DFD are often used to visualize a holistic vision of the system without going deep into detail.

\section{System dynamics (simulation)}

The systems dynamics approach models the nonlinear behavior of complex systems over time using stocks, flows, feedback loops, table functions and time delays. It reflects the structure of a system and the timeframe of interactions among its components. Mathematical modelling methods are employed.

2. deciding on the required and sufficient level of detail in the complex system representation;

3. choosing the language and format of element representation to express the content of the model.

The above difficulties make it impossible to use modelling software at the initial stage that of constructing a conceptual model. This produces the need to compare the research toolkit, the representation format and the level of complexity in the system with human capabilities. In order to address the task one needs to use to 
- use instruments that make it easier to systemically organize elements and links;

- present a super-complex system in an overview;

- pick an all-encompassing form of representing the content of the conceptual model.

Systemization instruments for elements and links. It is typical of a conceptual model to use the same structures for representing items that are different in nature. Such structures serve as a semantic framework - construct - for various items. Using multi-shaped constructs makes it much easier to identify and systemize system elements. Visualization of the construct helps to quickly classify elements when constructing a system and to set accents for better perception of the information. An example of construct utilization is shown in Fig. 1.

Overview. The intention to collect elements and see a subject as a single system is the main goal of constructing its model [4].

Using an overview simplifies the task of constructing and absorbing a conceptual model. An overview of a complex system is reflected in a well-picked metaphor. Information is arranged graphically, with the nature of elements and interconnections being conveyed through the key characteristics of the metaphor being used. The analysis of the model is guided by the interpretation of the metaphor. The approach enables one to focus on the essential elements and attributes of the system.

The list of metaphors that are used for organizing the elements of complex systems includes "tree", "metro map", "iceberg", "ruler" [16-19]. Some metaphors also serve as the basic constructs. For example, the "tree" metaphor could show the hierarchy in an organization and flows in a financial system. Figure 2 illustrates the use of the "matryoshka doll" metaphor for visualizing the architecture of the "Technology leadership incubator".

Form of expression. Depicting a conceptual model by means of verbal language only strongly narrows down opportunities for its examination and analysis. This is due to the fact that a verbal description of the diversity of its elements and their relationships will be as complex as the system itself. Other forms, too, have serious limitations because they use a certain vocabulary. It is possible to say that replacing one semiotic system with another affects the interpretation of the conceptual model and does not deliver the level of reflection that is needed in research. It is therefore necessary to use a language that offers a diversity of forms of expression: formulas, graphics, etc. An example of a combination of visual and verbal forms is given in Fig. 3.

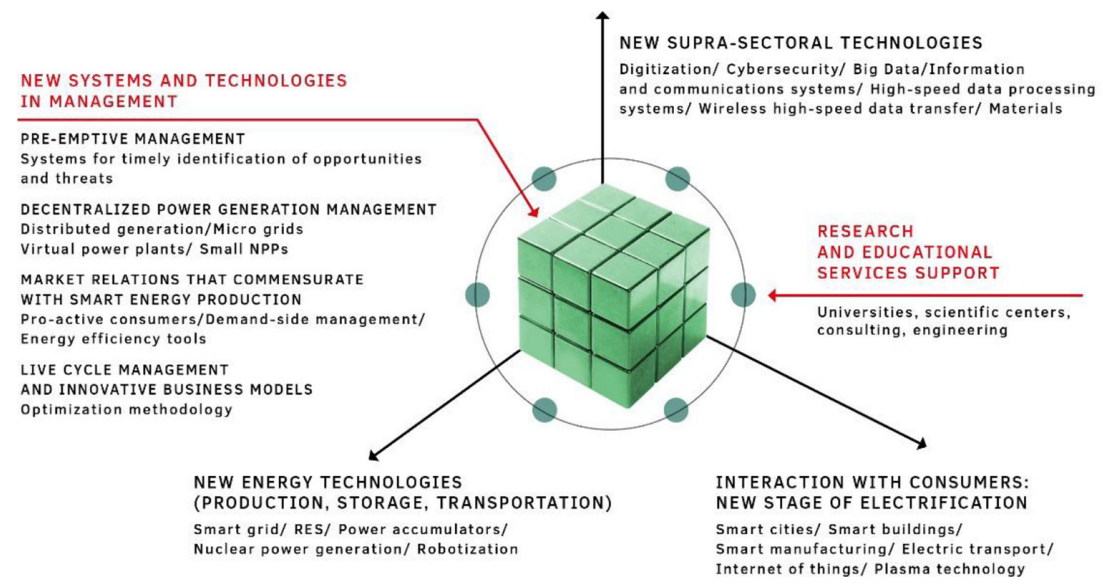

Figure 1: Construct utilization in the case of power engineering. 


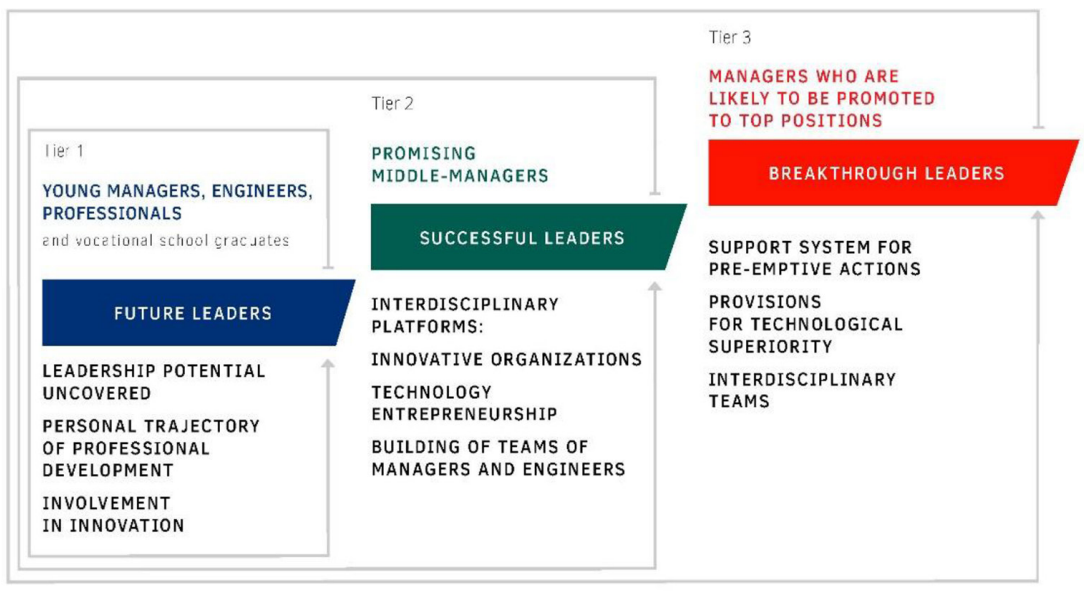

Figure 2: Metaphor utilization for producing an overview of the architecture of the Technology Leadership Incubator.

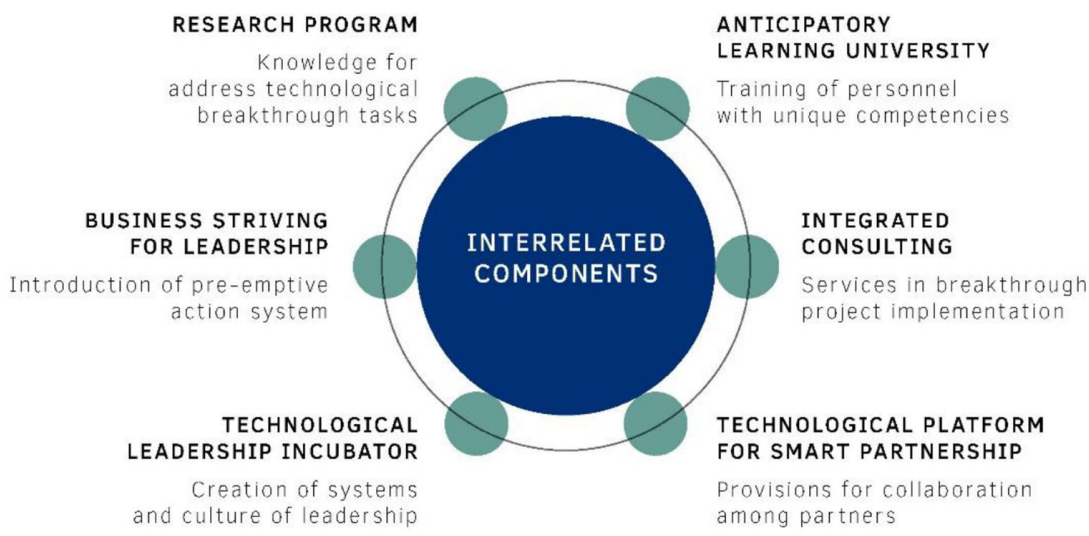

Figure 3: Combination of visual and verbal forms for graphic representation of the multiproject "New leaders for technological breakthrough".

The visualization method reveals the attributes that have the biggest importance for the purpose of research. It illustrates the features of the inner structure and the principle of relationships among the elements of a complex system, thus helping to propose ways of augmenting it.

\section{RESEARCH RESULTS}

The proposed method was tested as part of educational projects tailored for master's students and corporate customers of the Research and Educational Centre for Interdisciplinary Investigations and Educational Programs (REC ENGEC) at Ural Federal University in 2017-2018.

During organizational activity games, practitioners and university faculty members attended discussions of projects that aimed to address complex tasks of industrial modernization. Around 250 persons took part in special business games in 2017-2018. Some of the visual solutions to conceptual projects that were presented at the games are given below. 
Figure 4 shows the project of an online sales platform for the Novolipetsk Steel Works (NLMK Group), a leading vertically integrated steel manufacturer with assets in the US, Russia, France, Belgium, and Denmark. The integrated online sales platform show in the figure would provide for prompt interaction with end consumers, decrease delivery times, improve the flexibility and efficiency of capacity utilization thanks to control over the entire value chain.

Figure 5 shows a project aimed at the expansion of the utility market for T-Plus, the largest privately owned energy company in Russia. Today, the company only produces and sells heat

\section{IT-PLATFORM. WORK PRINCIPLES}
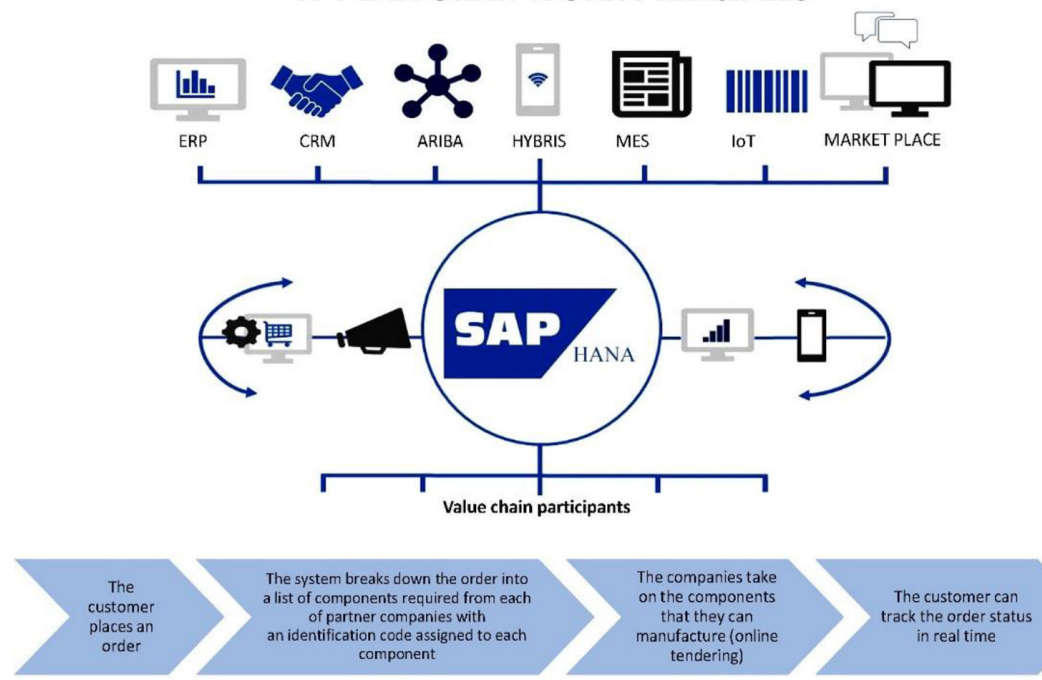

Figure 4: Online sales platform for NLMK Group

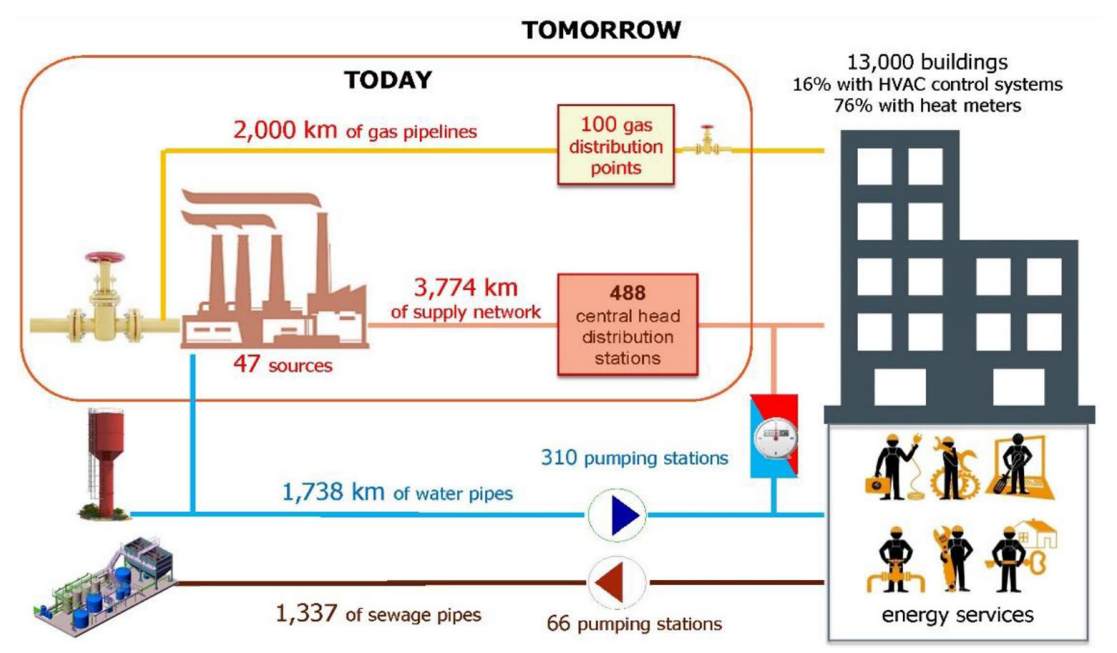

Figure 5: Expansion of T Plus utility market 


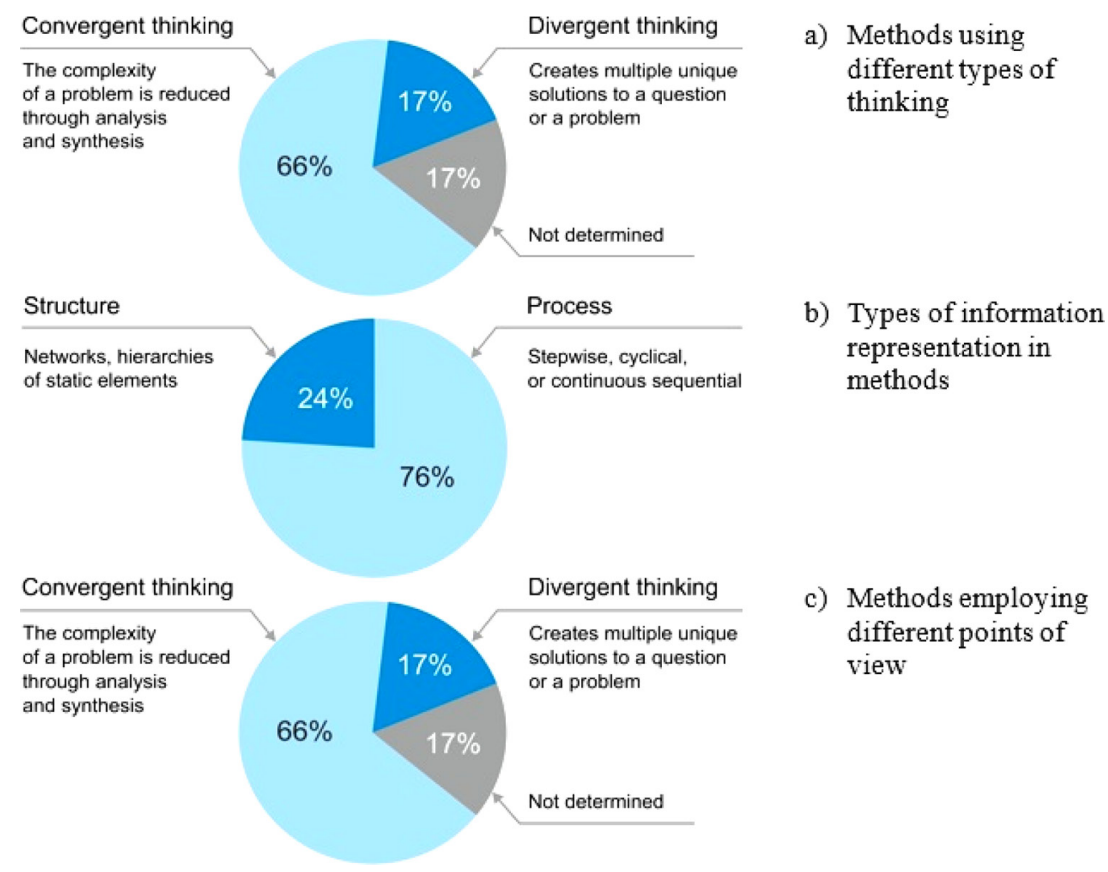

Figure 6: Distribution of visualization methods.

and electricity. It plans to acquire assets from companies with a similar profile to be able to provide maintenance services for HVAC control systems and house connections with the use of smart energy efficient technologies.

The results of practical application are backed with data produced by studies into the cognitive attributes of visualization methods [5]. The attributes could be divided into three groups: type of thinking; type of representation; and point of view. The percentage distribution of the attributes is found in Fig. 6.

\section{CONCLUSION}

Against the backdrop of accelerated digitization, convergence of markets and intrinsically difference systems, structures and development factors, visualization plays the role of an integrator that makes up for the absence of a unified conceptual framework and services as a "lighthouse", providing a clear map of priorities and targets for decision makers. It is therefore natural that the subject of visualization has started to attract growing attention both in science and in the areas of its application.

The novelty of this article lies in the fact that it investigates opportunities for visualization at the stages of complex systems design when no information is available yet about the ultimate parameters of the system, its interface and empirical characteristics. In this context, methods of visual analysis make it possible to test a hypothesis in terms of its overview and perception of the new system and to start a discussion in the multidisciplinary community of experts.

Professionals with visualization skills are needed for the successful adoption of visual language in complex systems management at the initial stage of the systems' lifecycle. At the moment, no such specialists can be found. This highlights the urgency of developing educa- 
tional programs that would nurture such competences. This would turn visual language into a natural form of interdisciplinary communication.

\section{ACKNOWLEDGEMENTS}

The work was supported by Act 211 of the Government of the Russian Federation, contract No 02.A03.21.0006.

\section{REFERENCES}

[1] Gitelman, L.D., Sandler, D.G., Gavrilova, T.B. \& Kozhevnikov, M.V., Complex systems management competency for technology modernization. International Journal of Design \& Nature and Ecodynamics, 12(4), pp. 525-537, 2017.

https://doi.org/10.2495/dne-v12-n4-525-537

[2] Gitelman, L.D., Sandler, D.G., Kozhevnikov, M.V. \& Tretyakov, V.S., Technology platform as a tool for transformation of university science and education activities, [in Russian]. University Management: Practice and Analysis, 4(98), pp. 31-42, 2015.

[3] Kress, G., Multimodality: A Social Semiotic Approach to Contemporary Communication, London: Routledge, pp. 212, 2010.

[4] Shneiderman, B., The eyes have it: a task by data type taxonomy for information visualizations. Proceedings of 1996 IEEE Conf. on Visual Languages (IV03), pp. 336-343, 1996.

https://doi.org/10.1109/vl.1996.545307

[5] Lengler, R. \& Eppler, M.J., Towards a periodic table of visualization methods for management. Proceedings of the IASTED International Conference on Graphics and Visualization in Engineering, pp. 83-88, 2007.

[6] Rzevski, G. \& Skobelev, P., Managing Complexity, Southampton, Boston: WIT Press, 2014, 198 p.

[7] Gavrilova, T.B., Gitelman, L.D. \& Kozhevnikov, M.V., System Engineering for Managers [in Russian]. Moscow: Economics, 2017, 188 p.

[8] Woods, D.D., Four concepts for resilience and the implications for the future of resilience engineering. Reliability Engineering and System Safety, 141, pp. 5-9, 2015. https://doi.org/10.1016/j.ress.2015.03.018

[9] Gillani, S.M., Qadri, S. \& Fahad M., Customer oriented requirement engineering by using scrum methodology. Internatıonal Journal of Natural and Engineering Sciences, 8(3), pp. 7-11, 2014.

[10] Barry, P. \& Dove, R., Combating uncertainty in the work flow of systems engineering projects. INCOSE International Symposium, 23(1), pp. 368-382, 2013.

[11] Barelkowski, R., Design process as complex system. International Journal of Design \& Nature and Ecodynamics, 13(1), pp. 46-59, 2018.

https://doi.org/10.2495/dne-v13-n1-46-59

[12] Meyer, R.E., Höllere, M.A., Jancsary, D. \& van Leeuwen, T., The visual dimension in organizing, organization, and organization research: Core ideas, current developments, and promising avenues. The Academy of Management Annals, 7(1), pp. 487-553, 2013. https://doi.org/10.1080/19416520.2013.781867

[13] Vittikh, V.A., Introduction to the Intersubjective Management Theory [in Russian], Samara: Samara Scientific Center of the Russian Academy of Sciences, 2013, 64 p.

[14] Horn, R.E., Visual Language: Global Communication for the 21st Century, Bainbridge, WA: MacroVU Press, 1998, 270 p. 
[15] Willems, K., The Periodic Table of Data Science. The 150+ companies, resources, and tools that define the data science industry, available at https://www.datacamp .com/community/blog/data-science-periodic-table?utm_campaign=crowdfire\&utm content=crowdfire \&utm_medium =social\&utm_source=instagram $\# 4542244293$ in\#1519033028315 (accessed 10 March 2018)

[16] Averbukh, V., Visualization metaphors. Programming and Computer Software, 27(5), pp. 227-237, 2001. https://doi.org/10.1023/a:1012333025189

[17] Risch, J.S., On the role of metaphor in information visualization, available at https:// arxiv.org/pdf/0809.0884.pdf (accessed 10 March 2018)

[18] Lima, M., Visualization metaphors: old \& new, available at https://www.interaliamag .org/articles/visualization-metaphors-old-new/ (accessed 10 March 2018).

[19] Ziemkiewicz, C. \& Kosara, R., The shaping of information by visual metaphors. IEEE Transactions on Visualization and Computer Graphics, 14(6), pp. 1269-1276, 2008. https://doi.org/10.1109/tvcg.2008.171 